\title{
An Extension of Generalized Cumulative Residual Entropy
}

\author{
Saeid Tahmasebi * \\ Department of Statistics, Persian Gulf University, Bushehr, Iran \\ tahmasebi@pgu.ac.ir \\ Maryam Eskandarzadeh \\ Department of Statistics, Persian Gulf University, Bushehr, Iran \\ eskandarymaryam@gmail.com
}

Ali Akbar Jafari

Department of Statistics, Yazd University, Iran

aajafari@yazd.ac.ir

Received 5 May 2015

Accepted 5 September 2016

\begin{abstract}
In this paper, a new extension of cumulative residual entropy is proposed. It contain the generalized cumulative residual entropy introduced by Psarrakos and Navarro (2013) and is related with the k-record values. We also consider a dynamic version of this new cumulative residual entropy using the residual lifetime. For these concepts, we obtain some properties similar to generalized cumulative residual entropy in stochastic ordering and aging classes properties.
\end{abstract}

Keywords: Cumulative residual entropy; Mean residual lifetime; Upper record values.

2000 Mathematics Subject Classification: 94A17, 62N05, 60E15

\section{Introduction}

Suppose $X$ denotes the lifetime of a system or living organism with probability density function (pdf) $f$. Shannon(1948) introduced a measure of uncertainty associated with $X$ as

$$
H(X)=-\int_{0}^{+\infty} f(x) \log f(x) d x
$$

Consider the lifetime $X$ of a system that has survived up to time $t$. In order to calculate the uncertainty about the residual life of such a system, Shannon entropy (1.1) is not appropriate. Ebrahimi

${ }^{*}$ Corresponding 
(1996) defined uncertainty of the residual lifetime of the random variable $X_{t}=[X-t \mid X>t]$ as

$$
H(X ; t)=H\left(X_{t}\right)=-\int_{t}^{+\infty} \frac{f(x)}{\bar{F}(t)} \log \frac{f(x)}{\bar{F}(t)} d x,
$$

where $\bar{F}(t)=\operatorname{Pr}(X>t)$ is the survival function of $X$. Recently, new measures of information are proposed in literature: replacing the pdf by the survival function in Shannon entropy, Rao et al. (2004) introduced a new measure of information that extends the Shannon entropy to continuous random variables, and called it cumulative residual entropy (CRE). The CRE is based on survival function $\bar{F}(x)$, and is defined as

$$
\mathscr{E}(X)=\int_{0}^{+\infty} \bar{F}(x) \Lambda(x) d x
$$

where $\Lambda(x)=-\log \bar{F}(x)$. Asadi and Zohrevand (2007) defined a dynamic version of the CRE (DCRE) by $\mathscr{E}(X ; t)=\mathscr{E}\left(X_{t}\right)$. Navarro et al. (2010) presented some stochastic ordering and aging classes properties of DCRE, and also defined the CRE and DCRE of past entropy $X_{[t]}=(t-X \mid X \leq$ $t$. Some new connections of the CRE and the residual lifetime are given by Kapodistria and Psarrakos (2012) using the relevation transform. Psarrakos and Navarro (2013) generalized the concept of CRE relating this concept with the mean time between record values and with the concept of relevation transform, and also considered dynamic version of this new measure. Raqab and Asadi (2010) obtained some mathematical properties of this generalized cumulative residual entropy (GCRE). Sunoj and Linu (2012) proposed the cumulative version of Renyi's entropy. Recently, Psarrakos and Toomaj (2017) obtained some results on generalized cumulative residual entropy with applications in actuarial science .

Let $\left\{X_{n}, n \geq 1\right\}$ be a sequence of independent and identically distributed random variables with cumulative distribution function (cdf) $F$ and pdf $f$. An observation $X_{j}$ will be called an upper record value if its value exceeds all previous observations. Thus, $X_{j}$ is an upper record value if $X_{j}>X_{i}$ for every $i<j$. For a fixed positive integer $k$, Dziubdziela and Kopocinski (1976) defined the sequence $\left\{U_{n(k)}, n \geq 1\right\}$ of $\mathrm{k}$-th upper record times for the sequence $\left\{X_{n}, n \geq 1\right\}$ as follows:

$$
U_{n(k)}=1, \quad U_{n+1(k)}=\min \left\{j>U_{n(k)}: X_{j: j+k-1}>X_{U_{n(k)}: U_{n(k)}+k-1}\right\}
$$

where $X_{j: m}$ denotes the $\mathrm{j}$-th order statistics in a sample of size $m$. Then $X_{n(k)}=X_{U_{n(k)}}$ is called a sequence of k-th upper record values of $\left\{X_{n}, n \geq 1\right\}$. The pdf of $X_{n(k)}$ is given by Dziubdziela and Kopocinski (1976) as follows:

$$
f_{n(k)}(x)=\frac{k^{n}}{(n-1) !}[\bar{F}(x)]^{k-1}[\Lambda(x)]^{n-1} f(x) .
$$

The cdf of Equation (1.4) can be obtained as

$$
F_{n(k)}(x)=\int_{0}^{x} \frac{k^{n}}{\Gamma(n)}[\bar{F}(y)]^{k-1}[\Lambda(y)]^{n-1} f(y) d y
$$




$$
=[\bar{F}(x)]^{k} \sum_{i=n}^{\infty} \frac{[k \Lambda(x)]^{i}}{i !}=1-[\bar{F}(x)]^{k} \sum_{i=0}^{n-1} \frac{[k \Lambda(x)]^{i}}{i !} .
$$

The above equation is obtained using the relationship between the incomplete gamma function and the sum of Poisson probabilities as follows:

$$
\int_{0}^{z} \frac{\lambda^{n}}{\Gamma(n)} x^{n-1} e^{-\lambda x} d x=\sum_{i=n}^{\infty} \frac{[\lambda z]^{i}}{i !} e^{-\lambda z}
$$

Hence, the survival function of $X_{n(k)}$ is given by

$$
\bar{F}_{n(k)}(x)=[\bar{F}(x)]^{k} \sum_{i=0}^{n-1} \frac{[k \Lambda(x)]^{i}}{i !}=w_{n}(\bar{F}(x)),
$$

where $w_{n}(x)=x^{k} \sum_{i=0}^{n-1} \frac{[-k \log x]^{i}}{i !}$ is an increasing function. Now, let $\mu_{n, k}(x)=\int_{0}^{+\infty} \bar{F}_{n(k)}(x) d x$. Then, from (1.7) we have

$$
k\left(\mu_{n+1, k}(x)-\mu_{n, k}(k)\right)=\int_{0}^{+\infty} \frac{k^{n+1}}{n !}[\bar{F}(x)]^{k}[\Lambda(x)]^{n} d x .
$$

Let $X$ be a non-negative absolutely continuous random variable with cdf $F$. Then similarly to the generalized cumulative residual entropy studied in Psarrakos and Navarro(2013), we can define an extension of CRE of $X$ as

$$
\begin{aligned}
\mathscr{E}_{n, k}(X) & =\int_{0}^{+\infty} \frac{k^{n+1}}{n !}[\bar{F}(x)]^{k}[\Lambda(x)]^{n} d x \\
& =\int_{0}^{+\infty} \frac{k^{n+1}}{n !}[\bar{F}(x)]^{k-1}[\Lambda(x)]^{n} f(x) \frac{\bar{F}(x)}{f(x)} d x \\
& =E_{X_{n+1(k)}}\left(\frac{1}{\lambda(X)}\right), \text { for } n=0,1,2, \ldots, k \geq 1,
\end{aligned}
$$

where $X_{n+1(k)}$ is a random variable with pdf (1.4) and $\lambda()=.\frac{f(.)}{\bar{F}(.)}$ is the hazard (failure) function of $F$. This new CRE is an extension of generalized CRE introduced by Psarrakos and Navarro(2013) and is obtained relating the concept of CRE with the mean time between $k$-record values. We call it extended cumulative residual entropy (ECRE). In this paper, we also consider the dynamic version of ECRE (DECRE) and obtain some properties of ECRE and DECRE.

This paper is organized as follows: In Section 2, we study some basic properties of ECRE. We also propose the dynamic version of ECRE, and obtain some results related to this measure. In Section 3, we state some relationships of ECRE with other concepts such as the mean lifetime of $X_{n(k)}$ and the mean residual lifetime of the random variable $\left[X_{n(k)}-t \mid X_{n(k)}>t\right]$.

\section{Some properties and characterization results}

In this section, we discuss some properties of ECRE. We also present a dynamic version of this concept with its characterization results. Here, $\leq_{s t}, \leq_{l r}$ and $\leq_{h r}$ denote the usual stochastic order, the likelihood ratio order and the hazard rate order, respectively. Also, the abbreviation for increasing (decreasing) failure rate, mean residual lifetime and decreasing (increasing) mean residual lifetime are IFR (DFR), MRL and DMRL (IMRL), respectively. 


\section{Proposition 2.1.}

i. If $k=1$, then the ECRE becomes to GCRE introduced by Psarrakos and Navarro(2013) i.e. $\mathscr{E}_{n, 1}(X)=\mathscr{E}_{n}(X)$.

ii. If $k=1$ and $n=1$, then the ECRE becomes to CRE introduced by Rao et al. (2004), i.e. $\mathscr{E}_{1,1}(X)=$ $\mathscr{E}(X)$.

iii. Let $X$ be a random variable of exponential distribution with mean $\lambda$, then we have $\mathscr{E}_{n, k}(X)=$ $\mathscr{E}_{n}(X)=\lambda$.

Proposition 2.2. If $X$ is IFR (DFR), then

$$
\mathscr{E}_{n, k}(X) \geq(\leq) \mathscr{E}_{n+1, k}(X), \text { for } n=1, \ldots, \quad k \geq 1 .
$$

Proof. Let $f_{n(k)}$ be the pdf of of $k$-record value $X_{n(k)}$. Then, the ratio $\frac{f_{n+1(k)}(t)}{f_{n(k)}(t)}=\frac{k}{n} \Lambda(t)$ is increasing in t. Therefore, $X_{n(k)} \leq_{l r} X_{n+1(k)}$, and this implies that $X_{n(k)} \leq_{s t} X_{n+1(k)}$, i.e.

$\bar{F}_{n(k)} \leq \bar{F}_{n+1(k)}$ (see Shaked and Shanthikumar 2007, Chapter 1). The proof is completed.

Theorem 2.1. If $X \leq_{S T} Y$ and $X$ is DFR, then

$$
\mathscr{E}_{n, k}(X) \leq \mathscr{E}_{n, k}(Y), \quad \text { for } n=1,2, \ldots, \quad k \geq 1 .
$$

Proof. It is well known that $X \leq_{H R} Y$ implies $X \leq_{S T} Y$ (see Shaked and Shanthikumar 2007). Hence from (1.7), we have

$$
\bar{F}_{n+1(k)}=w_{n+1(k)}(\bar{F}(t)) \leq w_{n+1(k)}(\bar{G}(t))=\bar{G}_{n+1(k)},
$$

where $\bar{G}_{n+1(k)}$ is the survival function of $Y_{n+1(k)}$. That is, $X_{n+1(k)} \leq_{S T} Y_{n+1(k)}$ holds. This is equivalent (see Shaked and Shanthikumar 2007, p.4) to have

$$
E\left(\phi\left(X_{n+1(k)}\right)\right) \leq E\left(\phi\left(Y_{n+1(k)}\right)\right)
$$

for all increasing functions $\phi$ such that these expectations exist. Thus, if we assume that $X$ is DFR and $\lambda(x)$ is its hazard rate, then $\frac{1}{\lambda(x)}$ is increasing and from (1.8), we have that

$$
\mathscr{E}_{n, k}(X)=E_{X_{n+1(k)}}\left(\frac{1}{\lambda(X)}\right) \leq E_{Y_{n+1(k)}}\left(\frac{1}{\lambda(X)}\right)
$$

On the other hand, $X \leq_{H R} Y$ implies that the respective hazard rate functions satisfy $\lambda(x) \geq \lambda(y)$. Hence, we have

$$
E_{Y_{n+1(k)}}\left(\frac{1}{\lambda(X)}\right) \leq E_{Y_{n+1(k)}}\left(\frac{1}{\lambda(Y)}\right)=\mathscr{E}_{n, k}(Y)
$$

Therefore, using both expressions we obtain $\mathscr{E}_{n, k}(X) \leq \mathscr{E}_{n, k}(Y)$.

Proposition 2.3. If $X$ is IFR (DFR), then

$$
\mathscr{E}_{n, k}(X) \leq(\geq) \mathscr{E}_{n, k+1}(X), \text { for } n=1,2, \ldots, \quad k \geq 1 .
$$

Proof. The proof is similar to Proposition 2.2. 
The dynamic version of the ECRE, that is, the ECRE of the residual lifetime $X_{t}=(X-t \mid X>t)$ is given by

$$
\mathscr{E}_{n, k}(X ; t)=\int_{t}^{+\infty} \frac{k^{n+1}}{n !}\left[\frac{\bar{F}(x)}{\bar{F}(t)}\right]^{k}\left[-\log \left(\frac{\bar{F}(x)}{\bar{F}(t)}\right)\right]^{n} d x, n=0,1,2, \ldots, k \geq 1
$$

This function is called dynamic extended cumulative residual entropy (DECRE). In the next proposition, we present some results of DECRE.

\section{Proposition 2.4.}

i. $\mathscr{E}_{n, k}(X ; 0)=\mathscr{E}_{n, k}(X)$.

ii. $\mathscr{E}_{0,1}(X ; t)=E\left(X_{t}\right)=m(t)$ is the MRL function of $X$.

iii. Let $X$ be a random variable of exponential distribution with mean $\lambda$, then we have

$$
\mathscr{E}_{n, k}(X ; t)=\mathscr{E}_{n-1, k}(X ; t)=\lambda .
$$

iv. If $X$ is $\operatorname{IFR}(D F R)$, then $\mathscr{E}_{n, k}(X ; t) \geq(\leq) \mathscr{E}_{n+1, k}(X ; t)$, for all $t$ and $n=1,2, \ldots$

v. If $X$ is $\operatorname{IFR}(D F R)$, then $\mathscr{E}_{n, k}(X ; t) \leq(\geq) \mathscr{E}_{n, k+1}(X ; t)$, for all $t$ and $k \geq 1$.

vi. By using the binomial expansion, we have

$$
\begin{aligned}
\mathscr{E}_{n, k}(X ; t) & =\int_{t}^{+\infty} \frac{k^{n+1}}{n !}\left[\frac{\bar{F}(x)}{\bar{F}(t)}\right]^{k}\left[-\log \left(\frac{\bar{F}(x)}{\bar{F}(t)}\right)\right]^{n} d x \\
& =\int_{t}^{+\infty} \frac{k^{n+1}}{n !}\left[\frac{\bar{F}(x)}{\bar{F}(t)}\right]^{k}[\Lambda(x)-\Lambda(t)]^{n} d x \\
& =\frac{k^{n+1}}{n ![\bar{F}(t)]^{k}} \int_{t}^{+\infty}[\bar{F}(x)]^{k} \sum_{i=0}^{n}\left(\begin{array}{c}
n \\
i
\end{array}\right)(-1)^{n-i}[\Lambda(x)]^{i}[\Lambda(t)]^{n-i} d x \\
& =\frac{k^{n+1}}{n ![\bar{F}(t)]^{k}} \sum_{i=0}^{n}\left(\begin{array}{c}
n \\
i
\end{array}\right)(-1)^{n-i}[\Lambda(t)]^{n-i} \int_{t}^{+\infty}[\bar{F}(x)]^{k}[\Lambda(x)]^{i} d x \\
& =\frac{k^{n+1}}{[\bar{F}(t)]^{k}} \sum_{i=0}^{n} \frac{(-1)^{n-i}}{i !(n-i) !}[\Lambda(t)]^{n-i} \int_{t}^{+\infty}[\bar{F}(x)]^{k}[\Lambda(x)]^{i} d x .
\end{aligned}
$$

vii.

$$
\begin{aligned}
\int_{t}^{+\infty}[\bar{F}(x)]^{k}[\Lambda(x)]^{n} d x & =n ![\bar{F}(t)]^{k} \mathscr{E}_{n, k}(X ; t) \\
& -\sum_{i=0}^{n-1}\left(\begin{array}{c}
n \\
i
\end{array}\right)(-1)^{n-i}[\Lambda(t)]^{n-i} \int_{t}^{+\infty}[\bar{F}(x)]^{k}[\Lambda(x)]^{i} d x
\end{aligned}
$$

viii.

$$
E\left[\mathscr{E}_{n, k}(X ; X)\right]=\int_{0}^{+\infty} \mathscr{E}_{n, k}(X ; x) f(x) d x
$$

In the following, we study some properties of aging classes and characterization results. To this purpose we first give an expression for the derivative of $\mathscr{E}_{n, k}(X ; t)$. 
Theorem 2.2. Let $X$ be a non-negative absolutely continuous random with $c d f F$, then

$$
\mathscr{E}_{n, k}(X ; t)=k \lambda(t)\left[\mathscr{E}_{n, k}(X ; t)-\mathscr{E}_{n-1, k}(X ; t)\right]
$$

Proof. The relation (2.4) can be written as

$$
\mathscr{E}_{n, k}(X ; t)[\bar{F}(t)]^{k}=k^{n+1} \sum_{i=0}^{n} \frac{(-1)^{n-i}}{i !(n-i) !}[\Lambda(t)]^{n-i} \int_{t}^{+\infty}[\bar{F}(x)]^{k}[\Lambda(x)]^{i} d x .
$$

By differentiating both sides with respect to $t$, we have

$$
\begin{aligned}
\mathscr{E}_{n, k}(X ; t)[\bar{F}(t)]^{k}-k f(t)[\bar{F}(t)]^{k-1} \mathscr{E}_{n, k}(X ; t) & =k^{n+1} \lambda(t) \sum_{i=0}^{n-1} \frac{(-1)^{n-i}}{i !(n-i-1) !}[\Lambda(t)]^{n-i-1} \int_{t}^{+\infty}[\bar{F}(x)]^{k}[\Lambda(x)]^{i} d x \\
& -k^{n+1}[\Lambda(t)]^{n}[\bar{F}(t)]^{k} \sum_{i=0}^{n} \frac{(-1)^{n-i}}{i !(n-i) !}
\end{aligned}
$$

Since

$$
\sum_{i=0}^{n} \frac{(-1)^{n-i}}{i !(n-i) !}=\frac{1}{n !} \sum_{i=0}^{n}(-1)^{n-i}\left(\begin{array}{c}
n \\
i
\end{array}\right)=0
$$

we conclude that

$$
\begin{aligned}
\mathscr{E}_{n, k}(X ; t)[\bar{F}(t)]^{k}-k f(t)[\bar{F}(t)]^{k-1} \mathscr{E}_{n, k}(X ; t) & =k^{n+1} \lambda(t) \sum_{i=0}^{n-1} \frac{(-1)^{n-i}}{i !(n-i-1) !}[\Lambda(t)]^{n-i-1} \\
& \times \int_{t}^{+\infty}[\bar{F}(x)]^{k}[\Lambda(x)]^{i} d x .
\end{aligned}
$$

Now by using (2.4), we have

$$
\mathscr{E}_{n, k}(X ; t)[\bar{F}(t)]^{k}-k f(t)[\bar{F}(t)]^{k-1} \mathscr{E}_{n, k}(X ; t)=-k \lambda(t)[\bar{F}(t)]^{k} \mathscr{E}_{n-1, k}(X ; t) .
$$

This completes the theorem.

Proposition 2.5. For $n, k=1$, we have the following relation which is given by Navarro et al.(2010) as

$$
\mathscr{\mathscr { E }}_{1,1}(X ; t)=\lambda(t)\left[\mathscr{E}_{1,1}(X ; t)-m(t)\right] .
$$

Theorem 2.3. If $X$ is IFR (DFR), then $\mathscr{E}_{n, k}(X ; t)$ is decreasing(increasing) for $n=0,1, \ldots$ and $k \geq 1$.

Proof. The result is true for $n=0$ and $k=1$ since $\mathscr{E}_{0,1}(X ; t)=m(t)$ is the MRL function of $X$ and it is well known that IFR (DFR) implies DMRL (IMRL). For $n \geq 1$ from Theorem 2.2, we have

$$
\mathscr{E}_{n, k}(X ; t)=k \lambda(t)\left[\mathscr{E}_{n, k}(X ; t)-\mathscr{E}_{n-1, k}(X ; t)\right] .
$$

Moreover, from (2.1), we have that if $X$ is IFR(DFR), then

$$
\mathscr{E}_{n, k}(X ; t) \leq(\geq) \mathscr{E}_{n-1, k}(X ; t) .
$$

Therefore, $\mathscr{E}_{n, k}(X ; t) \leq(\geq) 0$ for all $t$. Using this property we can define the following aging classes. 
Definition 2.1. We say that $X$ has an increasing (decreasing) DECRE of order $n$, shortly written as $I D E C R E_{n}\left(D D E C R E_{n}\right)$ if $\mathscr{E}_{n, k}(X ; t)$ is increasing (decreasing) in $t$.

Remark 2.1. i. If $X$ is IFR (DFR), then it is $D D E C R E_{n}\left(I D E C R E_{n}\right)$ for $n=0,1,2, \ldots$ and $k \geq 1$. ii. For $k=1, D D E C R E_{0}\left(I D E C R E_{0}\right)$ is equivalent to DMRL (IMRL).

Using again Theorem 2.2 we can obtain the following characterization result which extends the result obtained in Theorem 4.8 of Asadi and Zohrevand(2007).

Theorem 2.4. If for $k \geq 1 \mathscr{E}_{n, k}(X ; t)=c k \mathscr{E}_{n-1, k}(X ; t)$ holds for a fixed $n \in\{1,2, \ldots\}$ and $c>0$, then $X$ has a exponential $(c=1)$, a Pareto type II $(c>1)$ or a power distribution $(c<1)$.

Proof. This result was proved for $n=k=1$ in Theorem 4.8 of Asadi and Zohrevand(2007). Now, we assume that the result is true for $n-1$ and we are going to prove it for $n$. We are assuming that for $c>0$,

$$
\mathscr{E}_{n, k}(X ; t)=c k \mathscr{E}_{n-1, k}(X ; t)
$$

holds. Then the derivative of $\mathscr{E}_{n, k}(X ; t)$ is given by

$$
\mathscr{E}_{n, k}(X ; t)=c k \mathscr{E}_{n-1, k}(X ; t) .
$$

Moreover, from (2.6), we have

$$
\begin{aligned}
\mathscr{E}_{n, k}(X ; t)=c k \mathscr{E}_{n-1, k}(X ; t) & =k \lambda(t)\left[\mathscr{E}_{n, k}(X ; t)-\mathscr{E}_{n-1, k}(X ; t)\right] \\
& =k \lambda(t)(c k-1) \mathscr{E}_{n-1, k}(X ; t) .
\end{aligned}
$$

Analogously, using (2.6) for $n-1$, we get

$$
c k \mathscr{E}_{n-1, k}(X ; t)=c k^{2} \lambda(t)\left[\mathscr{E}_{n-1, k}(X ; t)-\mathscr{E}_{n-2, k}(X ; t)\right] .
$$

Therefore,

$$
\mathscr{E}_{n-1, k}(X ; t)=c k \mathscr{E}_{n-2, k}(X ; t),
$$

and hence, we get the stated result.

We have already mentioned that if $X$ is exponential, then $\mathscr{E}_{n, k}(X ; t)=\mathscr{E}_{n-1, k}(X ; t)=\ldots=m(t)=$ $\mu$. The preceding theorem proves that $\mathscr{E}_{n, k}(X ; t)=\mathscr{E}_{n-1, k}(X ; t)$ for a fixed $n$ and for all $t \geq 0$ characterizes the exponential model.

Proposition 2.6. $i$. Let $X$ be a non-negative random variable of Pareto type II model with survival function $\bar{F}(t)=\left[\frac{\beta}{t+\beta}\right]^{\alpha}(t \geq 0, \alpha, \beta>0)$, then we have $\mathscr{E}_{n, k}(X ; t)=c k \mathscr{E}_{n-1, k}(X ; t)=c^{n} k^{n+1} m_{k}(t)$, where $m_{k}(t)=\frac{t+\beta}{\alpha k-1}$ and $m_{k}(t) \leq \mathscr{E}_{1, k}(X ; t) \leq \mathscr{E}_{2, k}(X ; t) \leq \cdots \leq \mathscr{E}_{n, k}(X ; t)$. Hence, we obtian

$$
\mathscr{E}_{n, k}(X ; t)=\frac{\alpha^{n} k^{n+1}(t+\beta)}{(\alpha-1)^{n}(\alpha k-1)} .
$$

ii. Let $X$ be a non-negative random variable of power model with survival function $\bar{F}(t)=\frac{(\beta-t)^{\alpha}}{\beta^{\alpha}}$ $(0 \leq t<\beta, \alpha, \beta>0)$, then we have $\mathscr{E}_{n, k}(X ; t)=c k \mathscr{E}_{n-1, k}(X ; t)=c^{n} k^{n+1} m_{k}^{*}(t)$, where $m_{k}^{*}(t)=\frac{\beta-t}{\alpha k+1}$ and $m_{k}^{*}(t) \geq \mathscr{E}_{1, k}(X ; t) \geq \mathscr{E}_{2, k}(X ; t) \geq \cdots \geq \mathscr{E}_{n, k}(X ; t)$. Hence, we get

$$
\mathscr{E}_{n, k}(X ; t)=\frac{\alpha^{n} k^{n+1}(\beta-t)}{(\alpha+1)^{n}(\alpha k+1)} .
$$




\section{Relationships with other functions}

In this section, we state some relationships of $\mathscr{E}_{n, k}(X)$ with other functions. The proofs follow on the same line of Psarrakos and Navarro (2013).

Theorem 3.1. Let $X$ be a non-negative random variable with $c d f F$ and the hazard rate $\lambda(z), z>0$. Then for any $k \geq 1$ and $n=1,2, \ldots$, we have

$$
\mathscr{E}_{n, k}(X)=\frac{k^{n+1}}{n !} \int_{0}^{+\infty} \lambda(z)\left\{\int_{z}^{+\infty}[\bar{F}(x)]^{k}[\Lambda(x)]^{n-1} d x\right\} d z
$$

Proof. By (1.8) and the relation $\Lambda(x)=\int_{0}^{x} \lambda(z) d z$, we have

$$
\mathscr{E}_{n, k}(X)=\frac{k^{n+1}}{n !} \int_{0}^{+\infty} \int_{0}^{x} \lambda(z)[\bar{F}(x)]^{k}[\Lambda(x)]^{n-1} d z d x .
$$

With using Fubini's theorem, we get

$$
\mathscr{E}_{n, k}(X)=\frac{k^{n+1}}{n !} \int_{0}^{+\infty} \int_{z}^{+\infty} \lambda(z)[\bar{F}(x)]^{k}[\Lambda(x)]^{n-1} d x d z
$$

and the results follows.

Proposition 3.1. If $X$ is a continuous random variable with survival function $\bar{F}$, then we have the following result which is given in Psarrakos and Toomaj (2017) as

$$
\mathscr{E}_{n, 1}(X)=\frac{1}{n !} E\left[X(\Lambda(X))^{n}\right]-\frac{1}{(n-1) !} E\left[X(\Lambda(X))^{n-1}\right] .
$$

Theorem 3.2. If $X$ is a continuous random variable with $c d f F$, then for any $n=1,2, \ldots$, we have

$$
\begin{aligned}
\mathscr{E}_{n, k}(X) & =\frac{k^{n+1}}{n} E\left[\mathscr{E}_{n-1, k}(X ; X)(\bar{F}(X))^{k-1}\right] \\
& -\frac{k^{n+1}}{n !} \sum_{i=0}^{n-2}\left(\begin{array}{c}
n-1 \\
i
\end{array}\right)(-1)^{n-i-1} \int_{0}^{+\infty} \int_{z}^{+\infty} \lambda(z)[\Lambda(z)]^{n-i-1}[\bar{F}(x)]^{k}[\Lambda(x)]^{i} d x d z .
\end{aligned}
$$

Proof. Putting (2.5) in (3.1), we get

$$
\begin{aligned}
\mathscr{E}_{n, k}(X) & =\frac{k^{n+1}}{n !} \int_{0}^{+\infty} \lambda(z)(n-1) ![\bar{F}(z)]^{k} \mathscr{E}_{n-1, k}(X ; z) d z \\
& -\frac{k^{n+1}}{n !} \int_{0}^{+\infty} \lambda(z)\left[\sum_{i=0}^{n-2}\left(\begin{array}{c}
n-1 \\
i
\end{array}\right)(-1)^{n-i-1}[\Lambda(z)]^{n-i-1} \int_{z}^{+\infty}[\bar{F}(x)]^{k}[\Lambda(x)]^{i} d x\right] d z,
\end{aligned}
$$

or, equivalently

$$
\begin{aligned}
\mathscr{E}_{n, k}(X) & =\frac{k^{n+1}}{n} \int_{0}^{+\infty} \mathscr{E}_{n-1, k}(X ; z)[\bar{F}(z)]^{k-1} f(z) d z \\
& -\frac{k^{n+1}}{n !} \sum_{i=0}^{n-2}\left(\begin{array}{c}
n-1 \\
i
\end{array}\right)(-1)^{n-i-1} \int_{0}^{+\infty} \int_{z}^{+\infty} \lambda(z)[\Lambda(z)]^{n-i-1}[\bar{F}(x)]^{k}[\Lambda(x)]^{i} d x d z .
\end{aligned}
$$

The relation (3.1) completes the proof.

Bdair and Raqab(2012) defined the mean residual waiting time (MRWT) of the $(n+1)$ th shock time when the $n$th shock time did not fall in $(0, t)$. Specifically, 


$$
W_{n, k}(t)=E\left(X_{n(k)}-t \mid X_{n(k)}>t\right)=\frac{\sum_{j=0}^{n-1} \int_{t}^{+\infty} \frac{k^{j}}{j !}[\bar{F}(x)]^{k}[\Lambda(x)]^{j} d x}{\sum_{j=0}^{n-1} \frac{k^{j}}{j !}[\bar{F}(t)]^{k}[\Lambda(t)]^{j}} .
$$

A connection between $\mathscr{E}_{n, k}(X)$ and $W_{n, k}(t)$ is obtained in the following theorem.

Theorem 3.3. Let $X$ be a non-negative random variable with survival function $\bar{F}$, then for any $n=1,2, \ldots$, we have

$$
\mathscr{E}_{n, k}(X)=\frac{1}{n} \sum_{j=0}^{n-1}\left[k E\left[W_{n, k}\left(X_{(j+1) k}\right)\right]-j \mathscr{E}_{j, k}(X)\right]
$$

Proof. From relation (3.1), we obtain

$$
j \mathscr{E}_{j, k}(X)=\frac{k^{j+1}}{(j-1) !} \int_{0}^{+\infty} \lambda(z)\left\{\int_{z}^{+\infty}[\bar{F}(x)]^{k}[\Lambda(x)]^{j-1} d x\right\} d z
$$

Summing with respect to $j=1,2, \ldots, n$, we get

$$
\begin{aligned}
\sum_{j=1}^{n} j \mathscr{E}_{j, k}(X) & =\int_{0}^{+\infty} \lambda(z) \sum_{j=1}^{n} \int_{z}^{+\infty} \frac{k^{j+1}}{(j-1) !}[\bar{F}(x)]^{k}[\Lambda(x)]^{j-1} d x d z \\
& =\int_{0}^{+\infty} \lambda(z) \sum_{j=0}^{n-1} \int_{z}^{+\infty} \frac{k^{j+2}}{j !}[\bar{F}(x)]^{k}[\Lambda(x)]^{j} d x d z .
\end{aligned}
$$

Then, using (3.2), we have

$$
\begin{aligned}
\sum_{j=1}^{n} j \mathscr{E}_{j, k}(X) & =\int_{0}^{+\infty} \lambda(z) k^{2}\left[\sum_{j=0}^{n-1} \int_{z}^{+\infty} \frac{k^{j}}{j !}[\bar{F}(x)]^{k}[\Lambda(x)]^{j} d x\right] d z \\
& =\int_{0}^{+\infty} \lambda(z) k^{2} W_{n, k}(z)\left[\sum_{j=0}^{n-1} \frac{k^{j}}{j !}[\bar{F}(z)]^{k}[\Lambda(z)]^{j}\right] d z \\
& =\int_{0}^{+\infty} k W_{n, k}(z)\left[\sum_{j=0}^{n-1} \frac{k^{j+1}}{j !}[\bar{F}(z)]^{k-1}[\Lambda(z)]^{j} f(z)\right] d z \\
& =\int_{0}^{+\infty} k W_{n, k}(z) \sum_{j=0}^{n-1} f_{(j+1) k}(z) d z=\sum_{j=0}^{n-1} \int_{0}^{+\infty} k W_{n, k}(z) f_{(j+1) k}(z) d z \\
& =k \sum_{j=0}^{n-1} E\left[W_{n, k}\left(X_{(j+1) k}\right)\right] .
\end{aligned}
$$

Now, we can write

$$
\begin{aligned}
\sum_{j=1}^{n} j \mathscr{E}_{j, k}(X) & =\sum_{j=1}^{n-1} j \mathscr{E}_{j, k}(X)+n \mathscr{E}_{n, k}(X) \\
& =\sum_{j=0}^{n-1} j \mathscr{E}_{j, k}(X)+n \mathscr{E}_{n, k}(X)
\end{aligned}
$$




$$
=k \sum_{j=0}^{n-1} E\left[W_{n, k}\left(X_{(j+1) k}\right)\right] .
$$

So, we have

$$
\mathscr{E}_{n, k}(X)=\frac{1}{n} \sum_{j=0}^{n-1}\left[k E\left(W_{n, k}\left(X_{(j+1) k}\right)\right)-j \mathscr{E}_{j, k}(X)\right] .
$$

From (3.2), we can obtain the following result as

$$
W_{n, k}(t)=\sum_{j=0}^{n-1} M_{j, k}(t) p_{j, k}(t),
$$

where

$$
M_{j, k}(t)=\int_{t}^{+\infty} k^{j}\left[\frac{\bar{F}(x)}{\bar{F}(t)}\right]^{k}\left[\frac{\Lambda(x)}{\Lambda(t)}\right]^{j} d x,
$$

and

$$
p_{j, k}(t)=\frac{\frac{[\Lambda(t)]^{j}}{j !}}{\sum_{i=0}^{n-1} \frac{k^{i}[\Lambda(t)]^{i}}{i !}} .
$$

To get an useful connection between $W_{n, k}(t)$ and $\mathscr{E}_{n, k}(X ; t)$, we need the following lemma.

Lemma 3.1. Let $X$ be a non-negative random variable with survival function $\bar{F}$, then we have

$$
M_{j, k}(t)=\sum_{i=0}^{j} \frac{j !}{(j-i) !} \frac{k^{j-i-1}}{[\Lambda(t)]^{i}} \mathscr{E}_{i, k}(X ; t) .
$$

Proof. From (3.4), we have

$$
\begin{aligned}
M_{j, k}(t) & =\int_{t}^{+\infty} k^{j}\left[\frac{\bar{F}(x)}{\bar{F}(t)}\right]^{k}\left[\frac{\Lambda(x)}{\Lambda(t)}\right]^{j} d x \\
& =\int_{t}^{+\infty} k^{j}\left[\frac{\bar{F}(x)}{\bar{F}(t)}\right]^{k}\left[\frac{-\log \left(\frac{\bar{F}(x)}{\bar{F}(t)}\right)}{\Lambda(t)}+1\right]^{j} d x \\
& =\int_{t}^{+\infty} k^{j} \sum_{i=0}^{j}\left(\begin{array}{l}
j \\
i
\end{array}\right)\left[\frac{-\log \left(\frac{\bar{F}(x)}{\bar{F}(t)}\right)}{\Lambda(t)}\right]^{i}\left[\frac{\bar{F}(x)}{\bar{F}(t)}\right]^{k} d x \\
& =\sum_{i=0}^{j}\left(\begin{array}{l}
j \\
i
\end{array}\right) k^{j-i-1} \frac{1}{[\Lambda(t)]^{i}} \int_{t}^{+\infty} k^{i+1}\left[\frac{-\log \left(\frac{\bar{F}(x)}{\bar{F}(t)}\right)}{\Lambda(t)}\right]^{i}\left[\frac{\bar{F}(x)}{\bar{F}(t)}\right]^{k} d x \\
& =\sum_{i=0}^{j} \frac{j !}{(j-i) !} \frac{k^{j-i-1}}{[\Lambda(t)]^{i}} \int_{t}^{+\infty} \frac{k^{i+1}}{i !}\left[\frac{-\log \left(\frac{\bar{F}(x)}{\bar{F}(t)}\right)}{\Lambda(t)}\right]^{i}\left[\frac{\bar{F}(x)}{\bar{F}(t)}\right]^{k} d x \\
& =\sum_{i=0}^{j} \frac{j !}{(j-i) !} \frac{k^{j-i-1}}{[\Lambda(t)]^{i}} \mathscr{E}_{i, k}(X ; t) .
\end{aligned}
$$

Now we can obtain the connection between $W_{n, k}(t)$ and $\mathscr{E}_{n, k}(X ; t)$. 
Theorem 3.4. Let $X$ be a non-negative random variable with $c d f F$, then we have

$$
W_{n, k}(t)=\sum_{i=0}^{n-1} \mathscr{E}_{i, k}(X ; t) \gamma_{i, k}(t)
$$

where

$$
\gamma_{i, k}(t)=\frac{\sum_{j=0}^{n-i} \frac{k^{j-1}[\Lambda(t)]^{j}}{j !}}{\sum_{l=0}^{n-1} \frac{k^{l}[\Lambda(t)]^{l}}{l !}}, \quad i=0,1, \ldots, n
$$

Proof. By (3.3) and (3.4), we have

$$
\begin{aligned}
W_{n, k}(t) & =\sum_{j=0}^{n-1} \sum_{i=0}^{j} \frac{j !}{(j-i) !} \frac{k^{j-i-1}}{[\Lambda(t)]^{i}} \mathscr{E}_{i, k}(X ; t) p_{j, k}(t) \\
& =\sum_{i=0}^{n-1} \sum_{j=i}^{n-1} \frac{j !}{(j-i) !} \frac{k^{j-i-1}}{[\Lambda(t)]^{i}} \mathscr{E}_{i, k}(X ; t) p_{j, k}(t) \\
& =\sum_{i=0}^{n-1} \frac{\mathscr{E}_{i, k}(X ; t)}{[\Lambda(t)]^{i}} \sum_{j=i}^{n-1} \frac{j !}{(j-i) !} \frac{k^{j-i-1} \frac{[\Lambda(t)]^{j}}{j !}}{\sum_{l=0}^{n-1} \frac{k^{l}[\Lambda(t)]^{l}}{l !}} \\
& =\sum_{i=0}^{n-1} \mathscr{E}_{i, k}(X ; t) \frac{\sum_{j=i}^{n-1} \frac{k^{j-i-1}[\Lambda(t)]^{j-i}}{(j-i) !}}{\sum_{l=0}^{n-1} \frac{k^{l}[\Lambda(t)]^{l}}{l !}} \\
& =\sum_{i=0}^{n-1} \mathscr{E}_{i, k}(X ; t) \frac{\sum_{j=0}^{n-i} \frac{k^{j-1}[\Lambda(t)]^{j}}{j !}}{\sum_{l=0}^{n-1} \frac{k^{l}[\Lambda(t)]^{l}}{l !}} .
\end{aligned}
$$

Theorem 3.5. Let $X$ be a non-negative random variable with survival function $\bar{F}$, then for any $n=1,2, \ldots$, we have

$\mathscr{E}_{n, k}(X)=\frac{1}{n}\left\{\sum_{i=0}^{n-1} \frac{k^{i+2}}{i !} E\left([\bar{F}(X)]^{k-1}[\Lambda(X)]^{i} m_{n(k)}(X)\right)-\sum_{i=0}^{n-2} \frac{k^{i+2}}{i !} E\left([\bar{F}(X)]^{k-1}[\Lambda(X)]^{i} m_{n-1(k)}(X)\right)\right\}$,

where

$$
m_{n(k)}(t)=\frac{1}{\bar{F}_{n(k)}(t)} \int_{t}^{+\infty} \bar{F}_{n(k)}(x) d x, \quad n=1,2,3, \ldots
$$

is the mean residual lifetime of $X_{n(k)}$.

Proof. By (1.7), we see that

$$
\bar{F}_{n(k)}(t)-\bar{F}_{n-1(k)}(t)=\frac{[k \Lambda(t)]^{n-1}}{(n-1) !}[\bar{F}(t)]^{k} .
$$

Substituting the last equation in (3.1), we have

$$
\mathscr{E}_{n, k}(X)=\frac{k^{2}}{n} \int_{0}^{+\infty} \lambda(z)\left\{\int_{z}^{+\infty} \frac{[\bar{F}(x)]^{k}[k \Lambda(x)]^{n-1}}{(n-1) !} d x\right\} d z
$$




$$
\begin{aligned}
& =\frac{k^{2}}{n} \int_{0}^{+\infty} \lambda(z)\left\{\int_{z}^{+\infty}\left[\bar{F}_{n(k)}(x)-\bar{F}_{n-1(k)}(x)\right] d x\right\} d z \\
& =\frac{k^{2}}{n} \int_{0}^{+\infty} f(z)\left\{\frac{\bar{F}_{n(k)}(z)}{\bar{F}(z)} m_{n(k)}(z)-\frac{\bar{F}_{n-1(k)}(z)}{\bar{F}(z)} m_{n-1(k)}(z)\right\} d z \\
& =\frac{k^{2}}{n} \int_{0}^{+\infty} f(z)[\bar{F}(z)]^{k-1}\left\{\sum_{i=0}^{n-1} \frac{[k \Lambda(z)]^{i}}{i !} m_{n(k)}(z)-\sum_{i=0}^{n-2} \frac{[k \Lambda(z)]^{i}}{i !} m_{n-1(k)}(z)\right\} d z \\
& =\frac{1}{n} \sum_{i=0}^{n-1} \frac{1}{i !} \int_{0}^{+\infty} k^{i+2} f(z)[\bar{F}(z)]^{k-1}[\Lambda(z)]^{i} m_{n(k)}(z) d z \\
& -\frac{1}{n} \sum_{i=0}^{n-2} \frac{1}{i !} \int_{0}^{+\infty} k^{i+2} f(z)[\bar{F}(z)]^{k-1}[\Lambda(z)]^{i} m_{n-1(k)}(z) d z,
\end{aligned}
$$

and the result follows.

Remark 3.1. If $n=k=1$, then we have

$$
\mathscr{E}_{1,1}(X)=E(m(X)) .
$$

Note that some results of $m(X)$ are given by Asadi and Zohrevand (2007) and Novarro et al.(2010).

Proposition 3.2. Let $X$ be a non-negative random variable with $c d f F$, then for any $n=1,2, \ldots$, we have

$$
\mathscr{E}_{n, k}(X)=\frac{k}{n}\left\{\sum_{i=0}^{n-1} E\left(m_{n(k)}\left(X_{i+1}\right)\right)-\sum_{i=0}^{n-2} E\left(m_{n-1(k)}\left(X_{i+1}\right)\right)\right\},
$$

where $X_{i+1}$ is a random variable with $p d f f_{i+1(k)}$.

A generalization of the CRE by using the CRE of $X_{1: n}=\min \left(X_{1}, \ldots, X_{n}\right)$ is defined by

Baratpour (2010) as follows:

$$
\mathscr{E}\left(X_{1: n}\right)=-n \int_{0}^{+\infty}[\bar{F}(x)]^{n} \log \bar{F}(x) d x=n \int_{0}^{+\infty}[\bar{F}(x)]^{n} \Lambda(x) d x .
$$

Proposition 3.3. Let $X$ be a random variable of Pareto distribution with $p d f f(x)=\frac{\alpha \beta^{\alpha}}{x^{\alpha+1}}, x \geq \beta$. Then, it holds that

i. $\mathscr{E}(X)=\frac{\alpha \beta}{(\alpha-1)^{2}}$, and $\mathscr{E}\left(X_{1: n}\right)=\frac{n \alpha \beta}{(n \alpha-1)^{2}}$. Let $\Delta_{1}=\mathscr{E}(X)-\mathscr{E}\left(X_{1: n}\right)$, then Baratpour (2010) noted that for $\alpha>1, \Delta_{1} \geq 0$ i.e. the uncertainty of $X$ is bigger than that of $X_{1: n}$. Also for $n>\frac{1}{\alpha}, \Delta_{1}$ is an increasing function of $n$.

ii. $\mathscr{E}_{n, k}(X)=\frac{k a^{n} b}{(a k-1)^{n+1}}, a>\frac{1}{k}$, for $n=1,2, \ldots$ and $k \geq 1$. Note that $\mathscr{E}_{1,1}(X)=\mathscr{E}(X)$. Also, we obtain $\mathscr{E}_{n, k}(X)=\frac{a}{a k-1} \mathscr{E}_{n-1, k}(X)$, and $\mathscr{E}_{n, k}(X) \geq \mathscr{E}_{n-1, k}(X) \geq \ldots \geq \mathscr{E}_{1, k}(X)$. These results are expected since Pareto type I is a DFR distribution.

\section{Conclusions}

The ECRE proposed here contain the generalized cumulative residual entropy introduced by Psarrakos and Navarro (2013) and is related with the k-record values and with the relevation transforms. Also, a dynamic version of ECRE is considered. Some properties similar to generalized cumulative 
residual entropy in stochastic ordering and aging classes properties are obtained in this literature. These concepts can be applied in measuring the uncertainty contained in the associated residual lifetime.

\section{References}

[1] Asadi, M., Zohrevand, Y., 2007. On the dynamic cumulative residual entropy. J Stat Plan Inference. 137, 1931- 1941.

[2] Baratpour, S., 2010. Characterizations based on cumulative residual entropy of first-order statistics. Commun Stat Theory Methods. 39, 3645-3651.

[3] Bdair, O. M., Raqab, M. Z., 2012. Sharp upper bounds for the mean residual waiting time of records. Stat. 46(1), 69-84.

[4] Ebrahimi, N., 1996. How to measure uncertainty about residual life time. Sankhya Ser A, 58, 48-50.

[5] Dziubdziela, W., Kopocinski, B., 1976. Limiting properties of the k-th record value. Zastosowania Mathematyki. 15, 187-190.

[6] Kapodistria, S., Psarrakos, G., 2012. Some extensions of the residual lifetime and its connection to the cumulative residual entropy. Probability in the Engineering and Informational Sciences. 26, 129-146.

[7] Krakowski, M., 1973. The relevation transform and a generalization of the Gamma distribution function. Rev Francaise Autom Inf Res Oper. 7(2), 107-120.

[8] Navarro, J., del Aguila, Y., Asadi. M., 2010. Some new results on the cumulative residual entropy. J Stat Plan Inference. 140, 310-322.

[9] Psarrakos, G., Toomaj, A., 2017. On the generalized cumulative residual entropy with applications in actuarial science. Journal of Computational and Applied Mathematics. 309, 186-199.

[10] Psarrakos, G., Navarro, J., 2013. Generalized cumulative residual entropy and record values. Metrika. 76, 623-640.

[11] Rao, M., Chen, Y., Vemuri, B.C., Wang, F., 2004. Cumulative Residual Entropy: A New Measure of Information. IEEE Trans Inf Theory. 50(6), 1220-1228.

[12] Rao, M., 2005. More on a new concept of entropy and information. J Theor Probab. 18, 967-981.

[13] Raqab, M.Z., Asadi, M., 2010. Some results on the mean residual waiting time of records. Stat. 44, 493-504.

[14] Sunoj, S.M., Linu, M.N., 2012. Dynamic cumulative residual Renyis entropy. Stat. 46, 41-56.

[15] Shaked, M., Shanthikumar, J.G., 2007. Stochastic orders. Springer, New York.

[16] Shannon, C., 1948. A mathematical theory of communication. Bell System Technical Journal. 27, 379432. 